\title{
The Repairing of Concrete Walls in Structure for Water Treatment in Thermo Power Plant
}

\author{
Naser Kabashi, Cene Krasniqi and Ali Muriqi \\ Department of Civil Engineering, University of Prishtina, Prishtina 10000, Kosova
}

\begin{abstract}
As a result of longer term exploitation, exposure to severe weather conditions or influence of chemical conditions, concrete walls of concrete structures get damaged internally as well as externally. This study includes a preliminary analysis of chemically treated water in existing concrete structures, and another after the application of the method and using materials for the structures in future. One of the priorities was to examine the existing concrete structures by using nondestructive and destructive methods. After that, based on the results of the analysis, adequate new materials are proposed for the repairs, most commonly new technology polymer carbonated materials, in order to achieve durability of structure elements in using technological processes. Behavior of the repairing structures was tested using the in situ methods, and especially pull-of test, to verify the adhesion force between the old concrete structures and new applied layer. After the repairing, the concrete structures will be monitored to record the behavior under the chemical treated water.
\end{abstract}

Key words: Concrete structures, polymers, pull-of test, behavior under the treated water.

\section{Introduction}

More than half of the electricity generated in the world is by using coal as the primary fuel. Similarly, $90 \%$ of electrical power in Kosovo is generated in thermal power plants that operate using the coal. The function of the coal fired thermal power plant is to convert the energy available in the coal to electricity. Coal power plants work by using several steps to convert stored energy in coal to usable electricity that powers our lights, computers and sometimes, back into heat for our homes.

For properly work of power plants, it is necessary to cool the equipment on the tower.

Cooling water circuit-This part of the thermal power plant deals with the handling of the cooling water required by the system. Since the amount of water required to cool the outgoing steam from the boiler is substantial, it is either taken from a nearby water source such as a river, or done through

Corresponding author: Naser Kabashi, Dr., associate professor, research field: technology of concrete. E-mail: naser.kabashi@uni-pr.edu. evaporation if the quantity of cooling water available is limited [1, 2].

The process of cooling water is technological process using treated water in concrete structures-reactors.

\section{Effect of Treated Water in Concrete Structures}

One of the typical cooling systems is presented in Fig. 1, but treated water could be used as following:

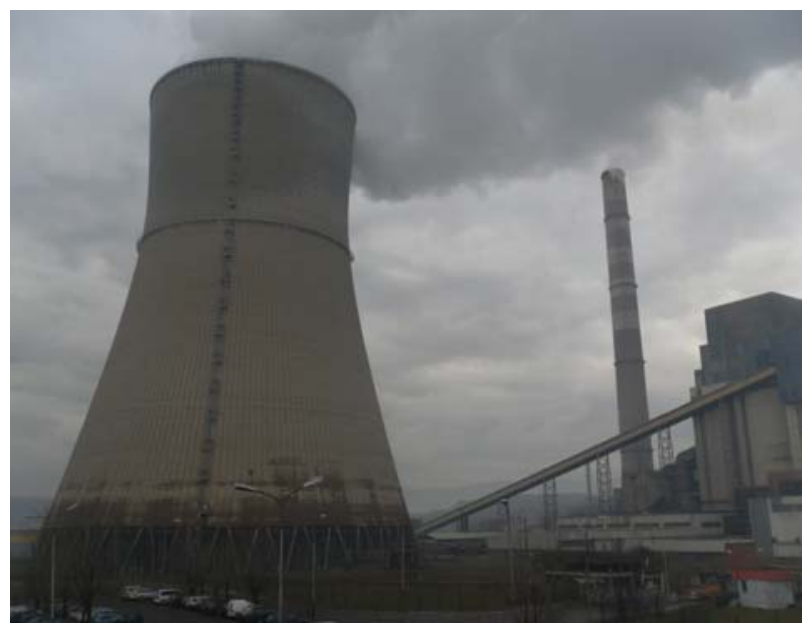

Fig. 1 Power thermal plant "KOSOVA-B". 
(1) Clarified water as cooling tower make-up and service water;

(2) De-mineralized water for heat cycle make-up, equipment cooling system makeup, condensate polishing plant regeneration etc.;

(3) Filtered and disinfected water for potable water requirement.

The water using for cooling system is treated water in two facilities:

(1) The treated water in water basins using the coagulant $\mathrm{Fe}_{2}\left(\mathrm{SO}_{4}\right)_{3}$ with polymers in percent of $48 \%$. In this way, the concentration in water will be reduced from 3\%-5\%;

(2) The next step will be treated with lime, whereby the concentration of hydrated lime is at $90 \%$, such the lime milk with dosage mass $170 \mathrm{mg} / \mathrm{L}$. The treated water will be rotated in reactors and after the homogenization will be used for cooling.
The practices alkalinity control of the cooling water, use acid neutralization (at two plants) or cold lime softening by precipitation (at six plants).

The long time of the technological process results with the concentration of the materials and is directly reflected in reactor concrete structures [3-5].

The situation of reactors is presented in Fig. 2 with the geometrical properties of structures [6].

\section{Concrete Structures under Several Conditions (under Treated Water)}

The concrete surface directly under the treated water was damaged on the surface as a result of different conditions. For this reason, an assessment is carried out applying the following steps [7, 8]:

(1) Visual inspection of structures;

(2) Examinations of quality of existing concrete;

(3) Cleaning and treating the surface;

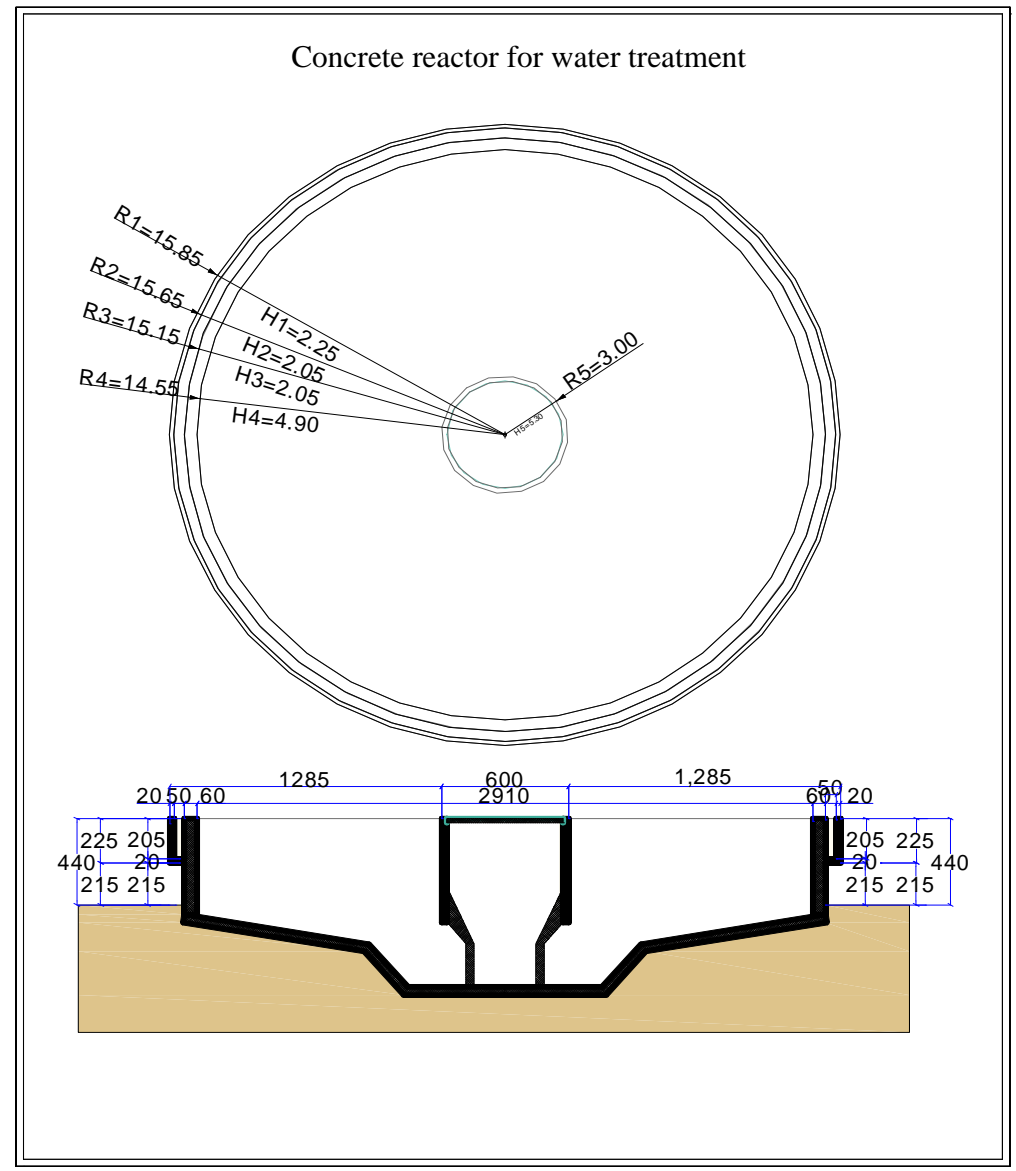

Fig. 2 Geometrical properties of concrete reactor. 

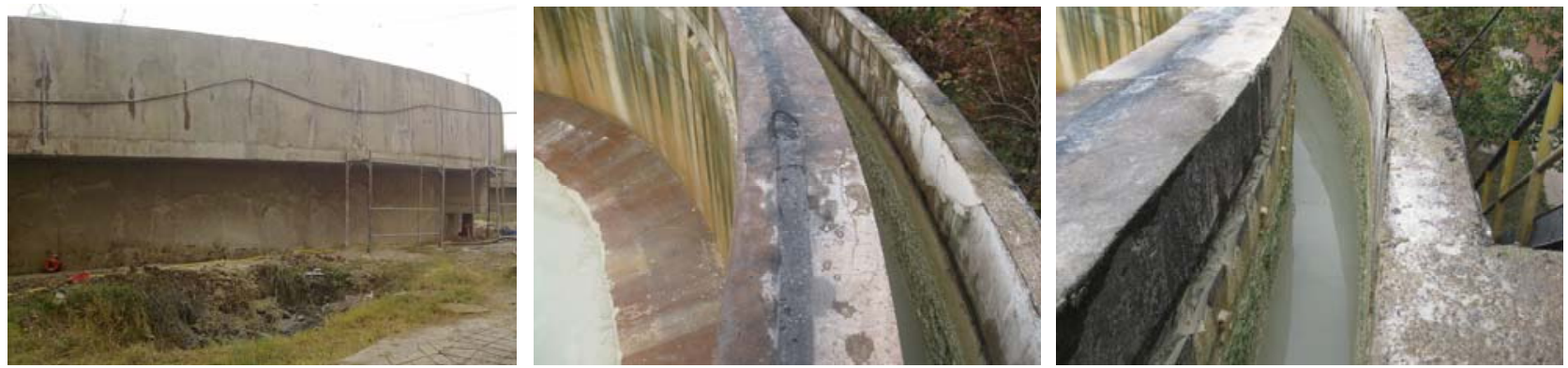

Fig. 3 The concrete structure during the visual inspection: outside and inside.

Table 1 Results of the analysis data of the concrete.

\begin{tabular}{llllllll}
\hline Pos & Date & Point & $X_{\min }$ & $X_{\max }$ & $s$ & $X_{\text {average }}$ & $\begin{array}{l}\text { Fck specified compressive } \\
\text { strength }\left(\mathrm{N} / \mathrm{mm}^{2}\right)\end{array}$ \\
\hline Wall & $>30 \mathrm{y}$ & $1-4$ & 41 & 55 & 3.6 & 61 & \\
Wall & $>30 \mathrm{y}$ & $5-8$ & 34 & 51 & 3.7 & 45.3 & \\
Wall & $>30 \mathrm{y}$ & $9-12$ & 39 & 50 & 3.8 & 46.1 & 49.2 \\
Wall & $>30 \mathrm{y}$ & $13-16$ & 46 & 54 & 2.3 & 50.9 & \\
Wall & $>30 \mathrm{y}$ & $17-20$ & 34 & 50 & 3.7 & 45.3 & \\
Wall & $>30 \mathrm{y}$ & $21-24$ & 42 & 52 & 2.9 & 48.1 & \\
\hline
\end{tabular}

(4) Materials for repairing the structures;

(5) Applying the methods for repairing;

(6) Repairs are evaluated through the examinations of structure.

\subsection{Visual Inspection of Existing Structures}

The visual inspection resulted in the need to remove the existing additional materials, because the process of damage of concrete surface is ongoing and also leaking water is also visible outside. The situation during the assessment is presented in Fig. 3.

\subsection{Examinations of Quality of Existing Concrete}

To analyze the quality of existing concrete, the authors applied the nondestructive examinations tests, in the first step of the preliminary results. The examinations were done in 20 different points with Hammer Schmidt in the perimeter of the concrete. The received data are presented in Table 1.

Based on the results, the quality of concrete allows to apply the repairing methods, including the injection methods and repairing the surface in both sides_-inside and outside.

\subsection{Cleaning and Treatment of the Surface}

The internal surface of concrete was damaged during as a result of 30 years of use and the sedimentation of the lime from treated water. The lime and sulphates are connected fast to the existing concrete and as a result both cleaning methods had to be applied:

(1) High pressure water for removing the damaged parts;

(2) Mechanical removal of the damaged part.

The surface before cleaning, during the cleaning and after cleaning is presented in Fig. 4 [7, 9-11].

The surface after the cleaning is treated with primer layer before applying the repairing mortar or layer of mortar.

\subsection{Materials for Repairing the Structures}

In this case the materials to be used for the repairs are analyzed based on the main properties:

(1) Behavior of the materials under the treated water;

(2) Compatibility of materials with existing concrete (quality of concrete);

(3) Durability of the repairing materials;

(4) Stopping the leaking of water outside of reactor.

The materials used are from the producer MAPEI-Italy: Primer, Mapefer, Mapegrout T40, 

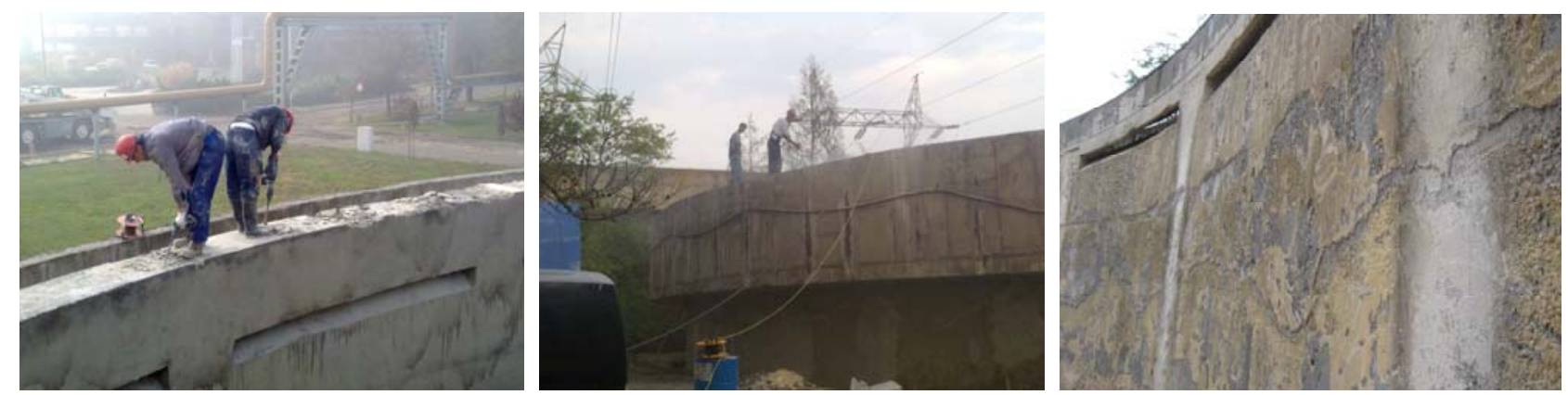

Fig. 4 Removal of the damaged parts and the cleaned concrete surface inside.

Mapelastic, Rexofoam $1 \mathrm{~km}$.

All the proposal materials meet the requested properties and are approved for repairing [9, 12].

\subsection{The Used Methods for Repairing}

The methods for repairing are: repair methods with mortar, injection methods and curing methods for repairing surfaces.

Different methods are applied for the following positions.

\subsubsection{Repairing Methods with Mortar}

Mortar Mapegrout T40 in technical specifications has the properties necessary for the wall repairing inside and outside.

Medium strength (40 MPa) fibre-reinforced thixotropic mortar is for the repair of concrete. Mapegrout T40 is also recommended for repair work in tunnels, canals and water works in general.

Mapegrout T40, mixed with about $16 \%$ water, forms a very workable mortar with a thixotropic consistency that is easily applied on vertical surfaces without shuttering. Repairs up to 30-35 mm thick in a

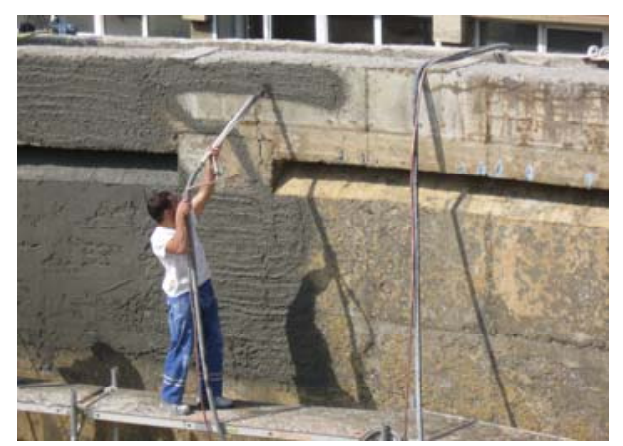

Fig. 5 Applying the mortar in prepared surfaces. single coat can be made.

To improve open-air curing and further reduce shrinkage, Mapegrout T40 can be mixed with 0.25\% by weight of Mapecure SRA, curing agent.

Mapegrout T40 meets the minimum requirements of EN 1504-3 standards for R3-class structural mortar $[9,12]$.

Fig. 5 presents the method of repairing surface using the dry methods of preparing mortar.

3.5.2 Injection Methods for Stopping Water Leakages

In the outside perimeter of concrete surface, there was a leakage of water in several spots. The method used is very adequate for this condition, because the space is enough for applying the necessary tools for repairing.

The application of these methods is presented in Fig. 6.

\subsection{Examinations of Repairs on Structures}

All activities will be under the EN Standard 1542, and must improve the durability of new repaired

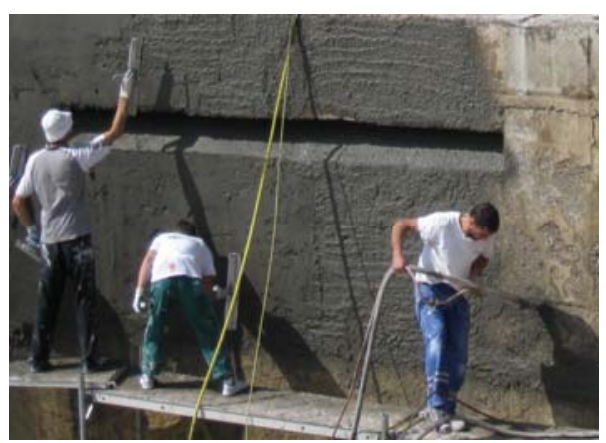



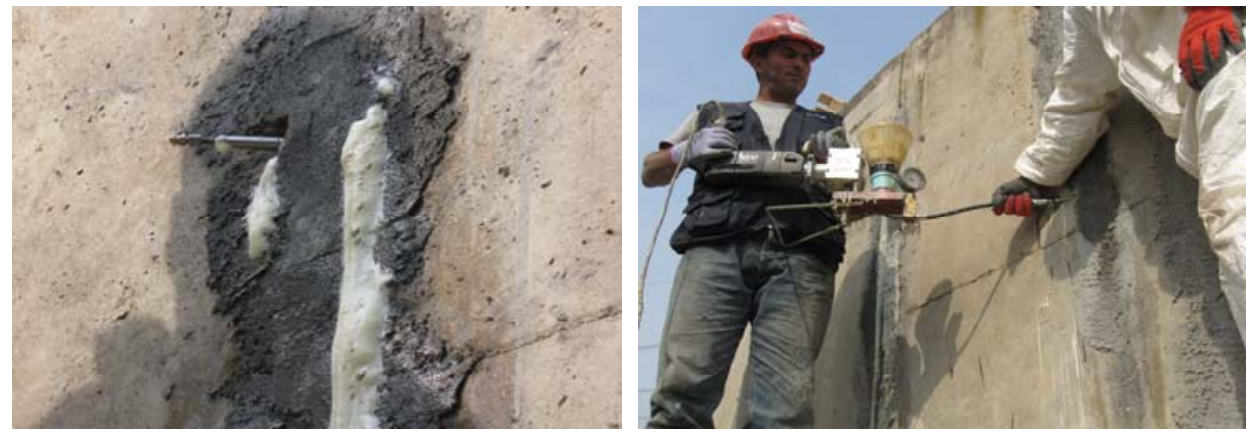

Fig. 6 The injection methods for stopping the water leaking from the reactor.
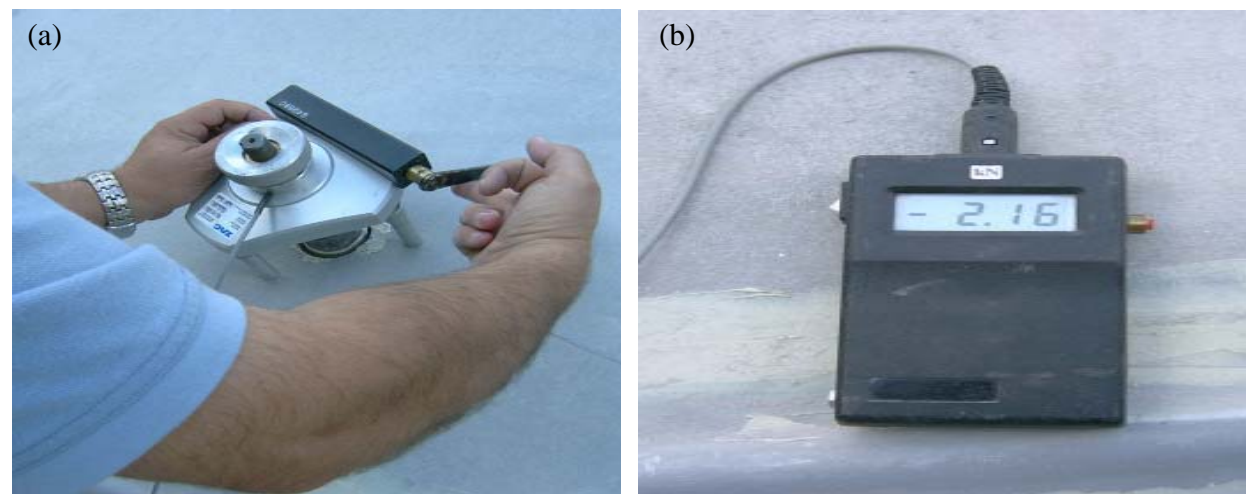

Fig. 7 The methodology of examinations and results: (a) examinations of bonding strength; (b) the results of bonding strength.

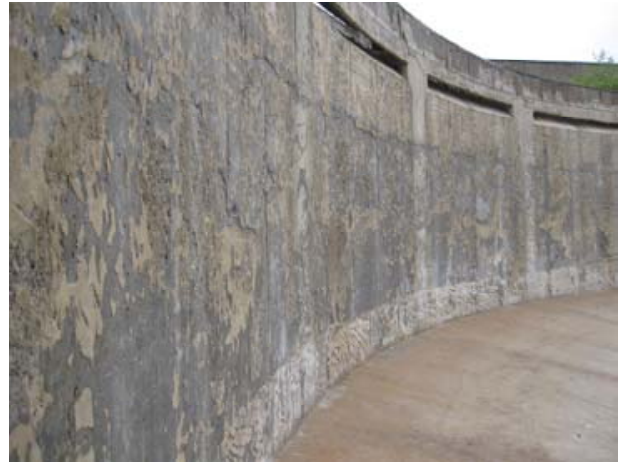

Fig. 8 Before repairing and after repairing.

structures. One of the requirements in this case is the bond strength between the old concrete and new layer.

To verify the bond strength, the authors used the "Pull-of Test Method-EN 1542" and the methodology of examinations and results are presented in Fig. 7 [7, 11-13], which situation before and after is presented in Fig. 8.

\section{Conclusions}

Based on the specifications of this project and in general for the repairing works, the authors can

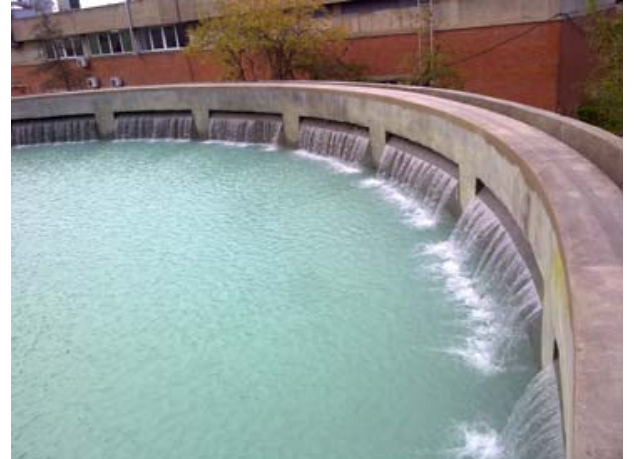

conclude:

- The treated water with chemical elements, environmental conditions and the indicative factors caused the damage in concrete structures and the authors' activity is focused for repairing the existing structure;

- The non-permanent assessment and non-permanent inspections, result on the concrete damaged under the specific conditions, such is treated water;

- Selection of repairing materials and methodology of repairs is decided after the analyzing the 
compatibility of the materials and the existing materials in structures;

- The testing results are satisfactory for this kind of structures and based on the preliminary request;

- The structure is in good conditions after the one year of repairing;

- Using the materials from the same producer is one of the good opportunity, in this case the materials from MAPEI-Italy have very good results.

\section{References}

[1] Case Study, Cooling Water Treatment in a Thermal Power Station, Degremont Technologies, Ozonia, 2002.

[2] Hamon Research Center, Hamon Wet Cooling Systems, German, 2011, pp. 2-7.

[3] B. Carney, A. McNemar, T. Feeley, Department of Energy/National Energy Technology Laboratory: Power Plant-Water R \& D Program, Department of Energy, USA, 2008.
[4] GEA Wiegand GmbH, Process Engineering: Thermal Treatment of Industrial Waste Water, the Bulletin on Energy Efficiency, German, 2011.

[5] Water Balance and Water Conservation in Thermal Power Stations, Bulletin of Energy Efficiency, USA, Dec. 2006.

[6] V.P. Eskom, Primary Energy (Water), Johannesburg, 2000.

[7] Concrete Repair Guide, ACI 546R-04, USA, 2004, p. 53.

[8] Causes, Evaluation and Repair of Reapproved 1998, Cracks in Concrete Structures, ACI 224.1R-93, USA, 1998.

[9] MAPEI-Products and Technical Data, Catalog of Products MAPEI-Manual, UK, 1973.

[10] B. Elsner, Corrosion inhibitors for steel in concrete, in: International Congress on Advanced Materials, Hawaii, 2000.

[11] P.H. Emmons, Concrete Repair and Maintenance, ACI Bookstore, USA, 1993.

[12] Pull of Test Method-EN 1542, MAPEI International, UK, 2005.

[13] Building Code Requirements for Structural Concrete, ACI 318-05, USA, 2011, p. 503. 